\title{
Factores de adherencia al tratamiento de hipertensión arterial en servicios de primer nivel de atención
}

\author{
Adherence factors to hypertension treatment in primary care services \\ Aicardo Solís ${ }^{1}$ orcid.org/0000-0001-6226-3541 \\ Gustavo Bergonzoli-Peláez2,3 orcid.org/0000-0001-9728-5329 \\ Adolfo Contreras-Rengifo4* orcid.org/0000-0002-0848-659X
}

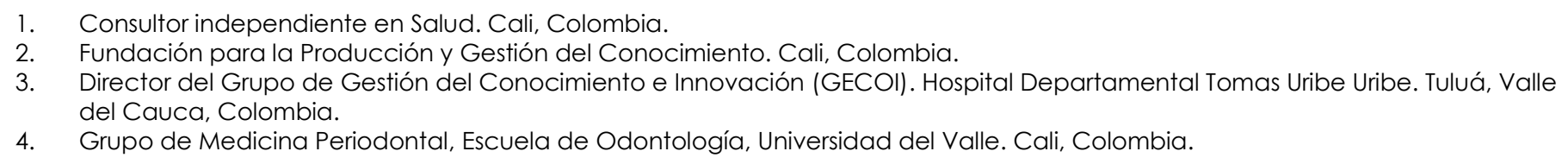

Fecha de recepción: Marzo 09 - $2020 \quad$ Fecha de revisión: Octubre 16 - $2020 \quad$ Fecha de aceptación: Diciembre 20 - 2021

Solís A, Bergonzoli-Peláez G, Contreras Rengifo A. Factores de adherencia al tratamiento de hipertensión arterial en servicios de primer nivel de atención. Univ. Salud. 2022;24(1):95-101. DOI: https://doi.org/10.22267/rus.222401.261

\section{Resumen}

Introducción: La enfermedad arterial hipertensiva no controlada (EAHNC), se asoció en 2008 con alta mortalidad por enfermedad cardiovascular que genera 9,4 millones de fallecimientos y 7\% de la carga de enfermedad expresada en Años de Vida Ajustados por Discapacidad (AVAD). Un 50\% de los pacientes no se adhieren al tratamiento EAH y se desconoce sobre las causas en el nivel básico de atención en Colombia. Objetivo: Identificar factores asociados con la adherencia al tratamiento de EAH en una Empresa Social del Estado (E.S.E), pública de un municipio colombiano. Materiales y métodos: Estudio de casos y controles, 75 casos y 75 controles seleccionados aleatoriamente a partir de una base de pacientes del programa de control de hipertensión arterial. Resultados: La edad mayor a 50 años fue el único factor asociado con falta de adherencia al tratamiento, sujetos entre 50-59 años con un OR=3,18 (Intervalo al 95\% de confianza) IC95\% 1,01-10,00; y entre 60-69 años 0R=3,70 IC95\% 1,17-11,60 tienen mayor probabilidad de no adherirse al tratamiento. Conclusiones: Los mayores de 50 a 69 años de edad presentaron la más alta probabilidad de no adherencia al tratamiento de la EAH. Se requieren reforzar medidas de seguimiento para mejorar su adherencia al tratamiento.

Palabras clave: Hipertensión; adherencia al tratamiento; estudios de casos y controles; regresión logística. (Fuente: DeCS, Bireme).

\section{Abstract}

Introduction: In 2008, uncontrolled hypertensive arterial disease (UHAD) was associated with a high cardiovascular mortality that caused 9.4 million deaths and 7\% of the disease burden expressed as Disability-Adjusted Life Years (DALYs). The reasons why 50\% of the patients treated in primary care facilities in Colombia do not adhere to UHAD treatment are unknown. Objective: To identify factors associated with UHAD treatment adherence in a Public Health Care institution from a Colombian city. Materials and methods: A case-control study with 75 cases and 75 controls randomly selected from a patient database of the arterial hypertension control program. Results: Being older than 50 years was the only factor associated with lack of adherence to treatment. Participants who were 50-59 (OR=3.18; IC95\% 1.01-10.00) and 60-69 (OR=3.70; IC95\% 1.17-11.60) are less likely to adhere to treatment. Conclusions: Patients who are 50-69 years old had the highest probability of non-adherence to UHAD treatment. Follow-up measures are necessary to improve this figure.

Keywords: Hypertension; treatment adherence and compliance; case-control studies; logistic models. (Source: DeCS, Bireme).

\footnotetext{
*Autor de correspondencia

Adolfo Contreras Rengifo

e-mail: adolfo.contreras@correounivalle.edu.co
} 


\section{Introducción}

Las enfermedades no transmisibles (ENT) representan una de las mayores amenazas para la salud, el desarrollo económico y social, especialmente en países en vías de desarrollo. Para controlar la epidemia de las ENT mundial se requiere intervenciones costo/efectivas que permitan aumentar la cobertura sanitaria entre aquellos sujetos que enfrentan barreras en el acceso, e incrementar la adherencia de los pacientes en tratamiento, toda vez que la enfermedad arterial hipertensiva es una afección crónica y silente, con complicaciones graves que ponen en riesgo la vida de los pacientes. La Organización Mundial de la Salud (OMS) recomienda aplicar intervenciones costo/efectivas de demostrada eficacia ya que significan para 2030, un retorno de US\$ 7 por persona por cada dólar invertido(1). Recientemente The Lancet Taskforce on NCDs and Economics, reveló una estrecha relación entre el crecimiento económico y el control de las enfermedades no transmisibles(2).

Según la OMS, la hipertensión, o tensión arterial alta, es un trastorno grave que incrementa de manera significativa el riesgo de sufrir cardiopatías, encefalopatías, nefropatías y otras enfermedades, como infarto agudo de miocardio, falla renal y pérdida de la visión. Se estima que a nivel mundial 1.130 millones sufren enfermedad arterial hipertensiva (EAH) y aproximadamente dos tercios, viven en países de ingresos bajos y medianos. Según las últimas proyecciones, la prevalencia de la EAH en adultos pasará de 918 millones en el año 2000 a 1,56 billones en 2025. En 2015, uno de cada cuatro hombres y una de cada cinco mujeres sufrían EAH y sólo un $20 \%$ de ellas estaban controladas. La hipertensión causa 9,4 millones de fallecimientos y el $7 \%$ de la carga de morbilidad -expresada en Años de Vida Ajustados por Discapacidad (AVAD), en 2010(2). La mayoría de las personas con EAH no presentan síntomas, por ello se le reconoce como el "asesino silencioso" y es la causa de por lo menos, un $45 \%$ de las muertes por cardiopatía isquémica y del 51\% de las muertes por accidente cerebrovascular. En 2014 la prevalencia mundial de la hipertensión en personas de 18 años o más fue de un $22 \%{ }^{(3,4)}$.

Se prevé que durante el periodo 2011-2025 la pérdida acumulada de producción asociada con las enfermedades no transmisibles en los países de ingresos bajos y medianos será de US\$ 7,28 billones (5). La pérdida anual de aproximadamente US\$
500,000 millones a causa de las principales enfermedades no transmisibles representa alrededor del $4 \%$ del producto interior bruto en esos países. Las enfermedades cardiovasculares, entre ellas la hipertensión, son responsables de alrededor de la mitad de dichos gastos( ${ }^{(6)}$.

La hipertensión es una de las principales causas de muerte prematura en el mundo, por ello, una de las metas mundiales contenidas en los Objetivos de Desarrollo Sostenible (ODS), es reducir la prevalencia de la hipertensión en un $25 \%$ para 2025 , con respecto a los valores de referencia de 2010(7).

En Colombia, según la Cuenta de Alto Costo, en junio de 2017, fueron reportados 3.776 .893 sujetos con diagnóstico de hipertensión arterial, con una prevalencia estimada de $7,7 \%$, lo que representa un incremento de 6,6\% respecto al 2016. El 61,6\% eran mujeres y el $62,5 \%$ pertenecían al régimen contributivo de seguridad social en salud. En general, la prevalencia ajustada por edad, es mayor entre las mujeres y muestra un incremento pasando de 7,5\% a $8,7 \%$ y de $5,3 \%$ a $6,4 \%$ entre los hombres ${ }^{(8)}$. Estas cifras son resultados de atenciones reportadas al Sistema de Alto Costo y no producto de encuestas poblacionales, por lo que se estima que la prevalencia sea mayor.

En el Municipio de La Cumbre (Valle del Cauca, Colombia), la Empresa Social del Estado (E.S.E.) Hospital Santa Margarita es el único hospital de atención básica. Durante el año 2015 reportó a través del perfil epidemiológico que la hipertensión arterial fue la primera causa de consulta, seguida de las complicaciones asociadas a factores cardiovasculares; es decir, el 19,6\% de la población mayor de 45 años atendida en la institución presenta Enfermedad Hipertensiva Crónica (EHC) ${ }^{(9)}$.

La E.S.E Hospital Santa Margarita, del municipio de La Cumbre, implementó el programa institucional de control de la hipertensión arterial, denominado Gestión de Riesgo Cardiovascular (GRC), que realiza intervenciones de tipo individual, familiar y colectivo, basadas en los principios de la estrategia de Atención Primaria en Salud, como eje central del modelo de atención de la E.S.E.

Dicho programa propone caracterizar el riesgo cardiovascular en la población mayor de 18 años, con el fin de establecer estrategias y acciones que permitan la reducción de las complicaciones 
asociadas a las ENT y la muerte prematura. A pesar de las acciones de diagnóstico, tratamiento, control y seguimiento, el $40 \%$ de los usuarios inscritos en el programa GRC son clasificados como no adheridos al mismo.

Por lo anterior, el objetivo de este estudio fue identificar los factores que facilitan o limitan la adherencia al tratamiento de la enfermedad arterial hipertensiva en los apacientes atendidos en la E.S.E Hospital Santa Margarita, del municipio de La Cumbre, Valle del Cauca, Colombia.

\section{Materiales y métodos}

\section{Tipo de estudio}

Se utilizó un estudio observacional, analítico, de casos y controles, tomando un control por cada caso. El marco muestral fueron los 743 pacientes inscritos en la base de datos del programa GRC, en el período comprendido entre enero y diciembre del 2016. Con base en los criterios: $P_{0}=0,40 ; 0 R=2,5 ; \alpha=0,05 y$ $\beta=0,20$ (10,11), se obtuvo una muestra de 75 casos y 75 controles. Se realizó un muestreo aleatorio sistemático, para la selección de los sujetos de estudio(12). La definición de no adherencia al tratamiento, se hizo con base en los siguientes criterios:

Casos: Todo paciente registrado en la base de datos del programa de control del riesgo cardiovascular de la institución de salud, con cifras de tensión arterial mayores o iguales a 140 sistólica y 90 diastólica, luego de 6 meses en tratamiento.

Controles: Se consideró adherido, a todo paciente registrado en la base de datos del programa de control del riesgo cardiovascular de la institución de salud, con cifras de tensión arterial inferiores a 139 de sistólica y 89 o menos de diastólica, luego de 6 meses en tratamiento.

\section{Recolección y control de calidad de la información}

Las variables incluidas en el análisis tuvieron como marco de referencia la revisión bibliográfica realizada y que, por supuesto, estuvieran registradas en la base de datos del programa de control de la institución sanitaria, con la finalidad de identificar los factores asociados más importantes en dicha región. Además, se recolectaron datos complementarios para medir variables potencialmente confusoras. Con el propósito de asegurar la calidad en la recolección de los datos, estos fueron digitados y revisados diariamente y semanalmente, se seleccionaron al azar un $10 \%$ de los datos. La información fue procesada y analizada con el software estadístico SPSS versión $24{ }^{\circledR} 10$.

\section{Plan de análisis}

Análisis exploratorio. Para el examen de las variables continuas se utilizó el promedio junto con la desviación estándar, en distribuciones sesgadas se optó por reportar la mediana y el rango intercuartílico. Para las variables con un número pequeño de categorías ordenadas (variables ordinales) se reportó las frecuencias y porcentajes.

En el análisis descriptivo de los datos, se empleó los estadísticos de tendencia central, dispersión y asimetría en variables cuantitativas medidas en escala de razón. Los gráficos de densidad y los diagramas de cajas y bigotes, se utilizaron para estudiar la distribución de las variables de tipo cuantitativo e identificar posibles valores atípicos. Las variables cualitativas se representan mediante el uso de tablas de frecuencia, diagramas de barras o de pastel que describen su valor modal. Se evaluó la presencia de números esperados menores de 5 en alguna de las celdas, cuando se presentó este fenómeno, se utilizó la prueba Exacta de Fisher, para evaluar la asociación, en otros casos el $\mathrm{Chi}^{2}$.

Se evaluó la normalidad de la distribución de las variables y se realizó la identificación los valores extremos que pudieran afectar la estabilidad de los estadísticos estimados (medidas de asociación). Se examinó la presencia de errores numéricos tales como la colinealidad establecida mediante el estadístico Factor de Inflación de la Varianza (FIV), la presencia de celdas con cero y la existencia de completa separación.

Análisis multivariado. Se realizó una selección de variables utilizando el procedimiento estadístico de eliminación paso a paso (Stepwise). También se incluyeron, en el modelo, variables que tuvieran importancia biológica o de salud pública, así no hubiesen arrojado significancia estadística en el análisis bivariado. Para la identificación de los factores potencialmente asociados con la adherencia o no, y dado que la variable respuesta estaba medida en una escala nominal dicotómica, se utilizó la regresión log-binomial. El Odds Ratio (OR) fue utilizado para estimar la fuerza de la asociación entre la variable respuesta y las variables independientes 
de interés en el estudio, ajustando por una serie de covariables que pudieran fungir como potenciales variables de confusión. La media de asociación OR se estimó tomando el antilogaritmo de cada uno de los coeficientes betas de las variables independientes arrojados por el modelo logístico, con su respectivo intervalo al $95 \%$ de confianza(13). Se realizó el diagnóstico del modelo obtenido, para esto se evaluó la bondad de ajuste del modelo logístico utilizando la prueba de Hosmer-Lemeshow ${ }^{(11-14)}$.

\section{Consideraciones éticas}

El proyecto contó con el aval del Comité de Ética para la Investigación con seres humanos de la Facultad de Salud - Universidad del Valle, código interno 065-015 de 2015.

\section{Resultados}

La variable edad fue la más cercana a la significancia estadística, razón por la cual se decidió crear estratos para ver si existía o no diferencias en cuanto a la adherencia, según diferentes grupos etarios (Tabla 1).

Tabla 1. Características socio-demográficas de los sujetos en estudio

\begin{tabular}{|c|c|c|c|c|c|}
\hline \multirow[b]{3}{*}{ Grupos de Edad } & \multicolumn{2}{|c|}{ Controles } & \multicolumn{2}{|c|}{ Casos } & \multirow[b]{2}{*}{$p^{*}$} \\
\hline & $n$ & $\%$ & $n$ & $\%$ & \\
\hline & & & & & \\
\hline Entre 31 y 49 años & 16 & 66,7 & 8 & 33,3 & 0,075 \\
\hline 50 o más años & 59 & $46,8 \%$ & 67 & 53,2 & \\
\hline \multicolumn{6}{|l|}{ Sexo } \\
\hline Mujer & 51 & 50,5 & 50 & 49,5 & 0,862 \\
\hline Hombre & 24 & 49,0 & 25 & $51,0 \%$ & \\
\hline \multicolumn{6}{|l|}{ Grupo étnico } \\
\hline Otra Etnia & 8 & 53,3 & 7 & 46,7 & 0,785 \\
\hline Mestizo & 67 & 49,6 & 68 & 50,4 & \\
\hline \multicolumn{6}{|l|}{ Estado civil } \\
\hline Unión libre & 34 & 48,6 & 36 & 51,4 & 0,913 \\
\hline Casado & 36 & 50,7 & 35 & 49,3 & \\
\hline Separado & 5 & 55,6 & 4 & 44,4 & \\
\hline \multicolumn{6}{|c|}{ Estrato socioeconómico } \\
\hline Bajo & 17 & 47,2 & 19 & 52,8 & 0,766 \\
\hline Medio & 34 & 48,6 & 36 & 51,4 & \\
\hline Alto & 24 & 54,5 & 20 & 45,5 & \\
\hline \multicolumn{6}{|l|}{ Régimen de salud } \\
\hline Contributivo & 7 & 41,2 & 10 & 58,8 & 0,440 \\
\hline Subsidiado & 68 & 51,1 & 65 & 48,9 & \\
\hline \multicolumn{6}{|l|}{ Aseguradora } \\
\hline Otra & 7 & 41,2 & 10 & 58,8 & 0,440 \\
\hline CafeSalud & 68 & 51,1 & 65 & 48,9 & \\
\hline \multicolumn{6}{|l|}{ Residencia } \\
\hline Urbano & 10 & 45,5 & 12 & 54,5 & 0,644 \\
\hline Rural & 65 & 50,8 & 63 & 49,2 & \\
\hline \multicolumn{6}{|l|}{ Asistió a remisión } \\
\hline No & 45 & 48,4 & 48 & 51,6 & 0,614 \\
\hline $\mathrm{Si}$ & 30 & 52,6 & 27 & 47,4 & \\
\hline
\end{tabular}

* Prueba $\mathrm{Chi}^{2}$ de Pearson

La prueba de la bondad del ajuste global del modelo no fue significativa, el valor de la probabilidad mayor a 5\%, nivel seleccionado como significante, por lo tanto, se acepta la hipótesis de que el modelo logístico es adecuado para examinar los datos disponibles (Tabla 2).
Tabla 2. Prueba de Hosmer-Lemeshow

\begin{tabular}{ccccc}
\hline Chí $^{2}$ & & gl & \multicolumn{2}{c}{ p-valor } \\
\hline & 7,25 & & 8 & 0,51 \\
\hline
\end{tabular}


Una vez estratificada la variable edad, se corrió el modelo logístico, incluyendo todas las variables analizadas, sólo la variable edad, estratificada arrojó significancia estadística. Los grupos entre 50 y 59 años con un $0 R=3,18$ y un IC95\% 1,01-10,0; y entre 60 y 69 años con un OR=3,70 y un IC95\% 1,17-11,6. Las demás variables no fueron significantes, por lo tanto, no parecen estar asociadas a la adherencia o no al tratamiento, en estos datos (Tabla 3).

Tabla 3. Factores asociados con la adherencia o no al tratamiento

\begin{tabular}{lrrr}
\hline & $\begin{array}{c}\text { Expo } \\
\text { (B) }\end{array}$ & \multicolumn{2}{c}{ I.C. 95\% para EXP(B) } \\
\cline { 2 - 4 } & Inferior & Superior \\
\hline Grupo de edad (Ref: Entre 30 y 49 años) & & \\
\hline 50-59 años & 3,2 & 1,01 & 10,01 \\
60-69 años & 3,7 & 1,17 & 11,61 \\
70-79 años & 2,5 & 0,732 & 8,570 \\
80 y más años & 2,6 & 0,588 & 11,709 \\
Sexo (Ref. Hombre) & 1,0 & 0,507 & 2,162 \\
Grupo étnico (Ref. & 1,3 & 0,405 & 4,273 \\
Mestizo) & & & \\
\hline Estado civil (Ref. Unión libre) & & & \\
\hline Casado & 0,8 & 0,406 & 1,685 \\
Separado & 0,6 & 0,139 & 2,740 \\
\hline Nivel Socioeconómico (Ref. Bajo) & & \\
\hline Medio & 1,1 & 0,480 & 2,962 \\
Alto & 0,7 & 0,289 & 2,017 \\
Régimen de salud (Ref. & 0,7 & 0,221 & 2,386 \\
Contributivo) & & & \\
Residencia (Ref. Urbano) & 0,9 & 0,344 & 2,570 \\
Tiempo de residencia & 0,9 & 0,964 & 1,016 \\
Tiempo en el programa & 0,7 & 0,359 & 1,718 \\
Asistió a remisión (Ref. & 0,7 & 0,378 & 1,597 \\
No) & & & \\
Constante & 1,542 & & \\
\hline
\end{tabular}

\section{Discusión}

El principal hallazgo de este estudio mostró que la ocurrencia de no adherencia aumenta con la edad, incrementándose tres veces más por cada 10 años de aumento, a partir de los 50 años. La no adherencia a la medicación es una creciente preocupación entre pacientes, médicos, sistemas de salud y otras partes interesadas porque es frecuente y se asocia con resultados adversos y mayores costos de atención. Aunque los métodos empleados en los estudios revisados no eran homogéneos, lo que puede explicar las diferencias en los resultados de las investigaciones; sin embargo, comúnmente se acepta que el incumplimiento de la adherencia por los hipertensos se sitúa en torno a 50-70 años de edad(15).
Un estudio realizado en Etiopía, reportó un resultado similar, con la edad de mayor riesgo para perder la adherencia al tratamiento(16). Un factor que reiterativamente se reporta en los estudios recientes, como factor de riesgo, es la edad, este hecho se puede deber a un efecto acumulativo de factores sociales a lo largo del curso de vida, o al proceso de envejecimiento o a ambos(16-18).

La adherencia a la terapia a largo plazo para enfermedades crónicas en los países desarrollados, presenta una amplia variación según sexo y país. En Canadá y Alemania, alcanza entre $50 \%$ y $58 \%$ en mujeres y entre $48 \%$ y $69 \%$ en hombres. En países como Irlanda, Japón, Italia, España y Finlandia la adherencia fluctúa entre $26 \%$ y $31 \%$ entre las mujeres y $17 \%$ y $26 \%$ entre los hombres. Algunos factores asociados con la menor adherencia se refieren a que las personas de mayor edad olvidan más la toma de los medicamentos, porque frecuentemente dichos enfermos padecen también otras afecciones, situación que implica la ingesta de múltiples medicamentos, lo cual incide en el abondo de estos. Por lo general, no hay un factor único que explique la no adherencia a la medicación, por tanto, debe adoptarse un enfoque integral para mejorarla, a partir del reconocimiento de los factores asociados con ella(17).

En Chile, un estudio reportó un 38,4\% de adherencia al tratamiento farmacológico y los factores positivamente asociados fueron: sexo masculino OR=1,54 IC95\% 1,23-1,93; síntomas de depresión $\mathrm{OR}=2,151,68-2,76$; bajo soporte social $\mathrm{OR}=1,52$ 1,22-1,90(18). Ninguno de estos factores fue identificado en el presente estudio.

En Suiza, reportaron que ser muy joven o muy mayor, bajo nivel educativo, pertenecer a un grupo étnico/racial marginado, bajo ingreso, poco apoyo social, mayores copagos en salud y residir en área rural, eran factores asociados con pobre adherencia al tratamiento de la hipertensión(19); el presente estudio coincide con la edad adulta.

En Tailandia, se encontró que solo el 12,8\% de los sujetos en estudio se podrían clasificar como adheridos al tratamiento farmacológico y el contar con una hija fue reportado como un factor que contribuía a la adherencia OR=7,99 IC85\% 1,25$53,7(20)$. 
En Suecia, un estudio evaluó la asociación entre el capital social, definido como el grado de interacción social entre los individuos de una familia/comunidad, y la gestión de la hipertensión en diferentes países. El principal hallazgo se relaciona con el aumento de la probabilidad de adherirse al tratamiento cuando se tiene capital social, este hallazgo sólo fue positivo en estratos de bajo ingreso, lo que, según los autores, podría estar significando una sustitución de la gestión de los servicios de salud, debido a su debilidad institucional(21).

Existe una experiencia de promoción de salud en Holanda, que utilizó la estrategia de dos tomas de presión arterial en casa, mediante el uso del instrumento Home Blood Pressure Mesaurement (HBPM) (por su sigla en inglés), que demostró ser adecuado en la identificación del $62,5 \%$ de los hipertensos que no sabían que lo eran. Este hallazgo es una evidencia a tener en cuenta, máxime que entre el $20 \%$ y $50 \%$ de las personas hipertensas, en la comunidad, desconocen su condición(22).

Otros hallazgos que se esperaban en relación a la literatura revisada sobre no adherencia(19-21), como el nivel socio económico, raza y sexo, no presentaron significancia estadística en este estudio, donde la población es socioeconómicamente similar por tratarse de un municipio relativamente rural y pobre.

\section{Limitaciones/fortalezas}

Comparar los resultados de este estudio con otros no es fácil, puesto que no siempre se utiliza la misma definición de caso, además de la omnipresente variación aleatoria. Este estudio, identificó algunos factores asociados a la adherencia al tratamiento de las variables registradas en la base de datos, y en ese sentido pueden haber quedado por fuera variables importantes en la adherencia.

Una fortaleza está dada por la ausencia del sesgo de memoria, definido como "el estímulo diferencial de la memoria"(23), puesto que los sujetos no fueron interrogados al usar los datos registrados en la base. El hecho de utilizar datos registrados por otras razones, generalmente administrativas, reduce también la probabilidad del sesgo de mala clasificación diferencial y en el peor escenario seria de mala clasificación no diferencial, cuya dirección hacia la hipótesis nula es reconocida.

Otra fortaleza es el control del sesgo de selección/participación y de no respuesta, pues se utilizó una muestra mediante un procedimiento aleatorio basada en todos los sujetos registrados en la base que tuvieran el $85 \%$ o más de los datos requeridos. De todas formas, es esperable la existencia de un sesgo residual, debido, principalmente, a la ausencia de algunas variables clave ausentes en la base de datos.

\section{Conclusiones}

Los pacientes de la E.S.E Hospital Santa Margarita mayores de 50 a 69 años de edad presentaron la más alta probabilidad de no adherencia al tratamiento de la EAH.

\section{Recomendaciones}

El enfoque de riesgo permitiría priorizar a los pacientes mayores de 50 años a través del trabajo que desarrollan los miembros del equipo de salud, especialmente los extramurales focalizados en la atención primaria en salud, con el fin de realizar seguimiento personalizado en casa, para evaluar el grado de la adherencia al tratamiento prescrito, dada la reconocida vulnerabilidad biológica y social de los pacientes mayores.

\section{Agradecimientos}

A la E.S.E Hospital Santa Margarita, del municipio de La Cumbre, a los pacientes que permitieron realizar este trabajo, a la Escuela de Salud Pública de la Universidad del Valle, por la asistencia técnica.

Conflicto de intereses: Ninguno declarado por los autores.

\section{Referencias}

1. Es Hora de Actuar: Informe de La Comisión Independiente de Alto Nivel de La OMS Sobre Enfermedades No Transmisibles. Ginebra: Organización Mundial de la Salud; 2018. Disponible en:

https://apps.who.int/iris/bitstream/handle/10665/27271 2/9789243514161-spa.pdf?ua=1

2. Lim SS, Vos T, Flaxman AD, Danaei G, Shibuya K, AdairRodahi $\mathrm{H}$, et al. A comparative risk assessment of burden of disease and injury attributable to 67 risk factors and risk factor clusters in 21 regions, 1990-2010: a systematic analysis for the Global Burden of Disease Study 2010. The Lancet. 2012;380(9859):2224-60. DOI: 10.1016/S01406736(12)61766-8.

3. Organización Mundial de la Salud. Informe Sobre La Situación Mundial de Las Enfermedades No Transmisibles 2014: "Cumplimiento de Las Nueve Metas Mundiales Relativas a Las Enfermedades No Transmisibles: Una Responsabilidad Compartida". Ginebra; 2014. 16 p. Disponible

$$
\text { en: }
$$


https://apps.who.int/iris/bitstream/handle/10665/14929 6/WHO_NMH_NVI_15.1_spa.pdf?sequence=1

4. Organización Mundial de la Salud. Información General Sobre a Hipertensión en el Mundo: Una Enfermedad Que Mata en Silencio, Una Crisis de Salud Pública Mundial; 2013. Disponible

en: https://apps.who.int/iris/bitstream/handle/10665/87679 /WHO_DCO_WHD_2013.2_spa.pdf;jsessionid=79F9E3F46F9 4214E22710B1701DCD9DA?sequence $=1$

5. World Health Organization, World Economic Forum. From Burden to "Best Buys ": Reducing the Economic Impact of Non-Communicable Diseases in Low- and Middle-Income Countries. Geneva; 2011. Disponible en: https://www.who.int/nmh/publications/best_buys_summa ry.pdf

6. Organización Panamericana de la Salud, Universidad de Washington. Las Dimensiones Economicas de Las Enfermedades No Transmisibles En America Latina y El Caribe. Washington D.C: Organización Mundial de la Salud; 2017. Disponible en: http://iris.paho.org/xmlui/bitstream/handle/123456789/ 33994/9789275319055-spa.pdf?sequence=1\&isAllowed=y

7. Organización Panamericana de la Salud. Salud En Las Américas+, Edición Del 2017. Resumen: Panorama Regional y Perfiles de País. Washington D.C: Organización Mundial de la Salud; 2017. Disponible en: https://www.paho.org/saluden-las-americas-2017/wp-

content/uploads/2017/09/Print-Version-Spanish.pdf

8. Fondo Colombiano de Enfermedades de Alto Costo. Situación La Enfermedad Renal Crónica, La Hipertensión Arterial y La Diabetes Mellitus En Colombia - 2018. Colombia; 2018. Disponible

en: https://cuentadealtocosto.org/site/publicaciones/situacion -de-la-enfermedad-renal-cronica-la-hipertension-arterial-yla-diabetes-mellitus-en-colombia-2018/

9. ESE Hospital Santa Margarita. Perfil Epidemiológico de La E.S.E Hospital Santa Margarita. Información Del Departamento de Estadística Relacionado Hipertensión Arterial y Factores Asociados. 2012.

10. IBM Corporation. IBM SPSS Statistics for Windows, Version 24.0. 2016. Disponible en: http://www01.ibm.com/support/docview.wss?uid=swg21476197

11. Schlesselman J. Case-Control Studies: Design, Conduct, Analysis. Oxford University Press; 1982. Disponible en: https://global.oup.com/academic/product/case-controlstudies-9780195029338?cc=co\&lang=en

12. Ryan T. Sample Size Determination and Power. En: John Wiley \& Sons, Inc. 2013. DOI: 10.1002/9781118439241.
13. Kleinbaum D, Klein M. Logistic Regression: A Self-Learning Text. 2nd ed. Springer; 2002. DOI: 10.1007/b97379.

14. Hosmer DW, Lemeshow S. Applied Logistic Regression. 2nd ed. En: John Wiley \& Sons, Inc. 2000. DOI: 10.1002/0471722146.

15. Prga I, Radman I, Leppée M, Culig J, Maric-Bajs M. The role of SMS reminders to increase the hypertensive patients' adherence to medication: Marcel Leppée. Eur J Public Health. 2016;26(Suppl 1):ckw174.111. DOI: 10.1093/eurpub/ckw174.111.

16. Teshome DF, Bekele KB, Habitu YA, Gelagay AA. Medication adherence and its associated factors among hypertensive patients attending the Debre Tabor General Hospital, northwest Ethiopia. Integr Blood Press Control. 2017;10:1-7. DOI: $10.2147 /$ IBPC.S128914.

17. NCD Risk Factor Collaboration (NCD-Risk). Long-term and recent trends in hypertension awareness, treatment, and control in 12 high-income countries: an analysis of 123 nationally representative surveys. The Lancet. 2019;394(10199):639-51. DOI: 10.1016/S01406736(19)31145-6.

18. Sandoval D, Nazzal C, Romero T. Clinical, Socioeconomic, and Psychosocial Factors Associated with Blood Pressure Control and Adherence: Results from a Multidisciplinary Cardiovascular National Program Providing Universal Coverage in a Developing Country. Int J Hypertens. 2018;2018:5634352. DOI: 10.1155/2018/5634352.

19. Burnier M, Egan BM. Adherence in Hypertension. Circ Res. 2019;124(7):1124-40. 10.1161/CIRCRESAHA.118.313220.

20. Woodham N, Taneepanichskul S, Somrongthong R, Auamkul $\mathrm{N}$. Medication adherence and associated factors among elderly hypertension patients with uncontrolled blood pressure in rural area, Northeast Thailand. J Heal Res. 2018;32(6):449-58. DOI: 10.1108/JHR-11-2018-085.

21. Palafox B, Goryakin Y, Suhrcke M, Balabanova D, Yusuf S, McKeeet M. Can social capital overcome barriers to effective management of hypertension. Study in 17 countries: Martin McKee. Eur J Public Health. 2016;26(Suppl 1):ckw164.068. DOI: 10.1093/eurpub/ckw164.068.

22. Niessen MAJ, van der Hoeven NV, van den Born B-JH, van Kalken CK, Kraaijenhagen RA. Home blood pressure measurement as a screening tool for hypertension in a webbased worksite health promotion programme. Eur J Public Health. 2014;24(5):776-81. DOI: 10.1093/eurpub/ckt144.

23. Bergonzoli G. Epidemiologia y Estadística: 1001 Preguntas [Lo Que No Se Atrevio a Preguntar, Pero Siempre Quiso Saber]. IMPRETICS E.I.C.E. 2019. 